\title{
Performance of HPV DNA testing in the follow-up after treatment of high-grade cervical lesions, adenocarcinoma in situ (AIS) and microinvasive carcinoma
}

\author{
Silvano Costa ${ }^{1^{*}}$, Simona Venturoli ${ }^{2}$, Massimo Origoni ${ }^{3^{*}}$, Mario Preti ${ }^{4^{*}}$, Luciano Mariani ${ }^{5^{*}}$, Paolo Cristoforoni ${ }^{6^{*}}$ and \\ Maria Teresa Sandri ${ }^{*}$
}

\begin{abstract}
'Obstetrics \& Gynaecology Unit, Policlinico S Orsola-Malpighi University Hospital, Bologna, Italy Present address: MF Toniolo Hospital, via Toscana, 42, Bologna 40138, Italy

${ }^{2}$ Unit of Microbiology, Department of Diagnostic Medicine and Prevention, S Orsola-Malpighi Hospital, University of Bologna, Bologna 40138, Italy

${ }^{3}$ Department of Obstetrics \& Gynaecology, School of Medicine, Vita-Salute San Raffaele University, Milano 20132, Italy

${ }^{4}$ Preventive Gynaecology Unit, European Institute of Oncology, Milano 20141, Italy

${ }^{5} \mathrm{HPV}$ Unit, Gynaecologic Oncology, Regina Elena National Cancer Institute of Rome, Rome 00144, Italy

${ }^{6}$ National Institute on Cancer Research (IST), Genova 16132, Italy
\end{abstract}

*The Italian HPV Study Group (IHSG)

Correspondence to: Silvano Costa. Email: costa.silvano@libero.it

\section{Abstract}

Background: Over the last two decades it has become clear that distinct types of human papillomavirus (HPV), the so-called high-risk types (hrHPV), are the major cause of cervical cancer. The hrHPV-DNA testing has shown excellent performance in several clinical applications from screening to the follow-up of conservatively treated patients.

Methods: We conducted a systematic review of the recent literature on the performance of HPV DNA testing in follow-up after treatment of high-grade cervical lesions, adenocarcinoma in situ, and microinvasive carcinoma compared to Pap smear cytology.

Results: Observational studies have demonstrated that the high risk hrHPV-DNA test is significantly more sensitive (95\%) compared to follow-up cytology $(70 \%)$ in detecting post-treatment squamous intraepithelial high-grade lesions. Moreover, in patients treated conservatively for cervical adenocarcinoma in situ, the hrHPV-DNA test is the most significant independent predictor of recurrent disease or progression to invasive cancer, and the combination of viral DNA testing and cytology reaches $90 \%$ sensitivity in detecting persistent lesions at the first

Published: 29/04/2015

Received: 18/12/2014

ecancer 2015, 9:528 DOI: 10.3332/ecancer.2015.528

Copyright: (c) the authors; licensee ecancermedicalscience. This is an Open Access article distributed under the terms of the Creative Commons Attribution License (http://creativecommons.org/licenses/by/3.0), which permits unrestricted use, distribution, and reproduction in any medium, provided the original work is properly cited. 
follow-up visit and $100 \%$ at the second follow-up visit. The cause of microinvasive squamous cervical carcinoma is increasingly treated with conservative therapies in order to preserve fertility, and an effective strategy allowing early detection of residual or progressive disease has become more and more important in post-treatment follow-up. Primary results seem to indicate that the median time for viral clearance is relatively longer compared with patients treated for $\mathrm{CIN}$ and suggest a prolonged surveillance for these patients. However, the potential clinical value of HPV-DNA testing in this clinical setting needs to be confirmed by further observations.

Conclusions: The excellent sensitivity, negative predictive value, and optimal reproducibility of the hrHPV DNA testing, currently is considered a powerful tool in the clinicians' hands to better manage post-treatment follow-up either in cervical squamous lesion or in situ adenocarcinoma.

Keywords: CIN recurrence, HPV-testing, genotyping, CIN2+ lesion, adenocarcinioma in situ, microinvasive squamous carcinoma, follow-up

\section{Introduction}

Several types of cervical surgical procedures are used to treat cervical squamous intraepithelial neoplasia (CIN2-3) and in selected cases of adenocarcinoma in situ (AIS), such as loop electrosurgical excision procedure (LEEP) or conisation using a laser knife or cold-knife [1]. Nevertheless, the long-term risk of CIN persistence/recurrence or invasive carcinoma remains higher among women who undergo conservative treatment and they require continued surveillance and follow-up. In particular, between $5 \%$ and $20 \%$ of cases develop recurrent disease within three years and the risk of invasive cervical cancer is still five-fold greater than that in the general population [2-7]. A recent paper estimated that in the UK $16 \%$ of diagnosed cervical cancers had previously been treated for intraepithelial neoplasia $[6,7]$. The main reasons include residual disease because of incomplete removal of the primary lesion and post-treatment persistent infection of hrHPV [8].

Currently, despite it's low sensitivity [1, 2], the Papanicolaou (Pap) test is widely used in the follow-up of patients treated for intraepithelial neoplasia and the European guidelines for cervical screening policy recommends 6-, 12-, and 24-month cytology after CIN treatment [9].

\section{HPV DNA testing}

A large amount of published data over recent decades on HPV DNA testing by means of pooled-based or type-specific methods has definitely demonstrated that persistent positivity of viral infection is considered a prognostic index of persistent/recurrent disease in patients treated for CIN2-3/ AIS. Observational studies demonstrated that post-treatment positive HPV-DNA test predicts residual/recurrent CIN with a significantly higher sensitivity and a non-significantly lower specificity than conventional cytology-based follow-up, $95 \%$ versus $70 \%$ and $75 \%$ versus $78 \%$ respectively. Overall, combined hrHPV and cytology testing yielded the best performance; the combination hrHPV-DNA testing and cytology demonstrated a $96 \%$ sensitivity, $81 \%$ specificity (Table 1) with $99 \%$ of negative predictive value (NPV) [1-3, 7, 10, 13, 15, 19]. Based on these considerations the American Society of Colposcopy and Cervical Pathology (ASCCP) recommends the use of HPV testing together with cytology (co-testing) at 12 and 24 months following treatment for CIN2-3 [11].

Table 1. Performance of high-risk hrHPV-DNA, cytology, and cotesting for cytology and hrHPV DNA in predicting residual/recurrent disease after conservative therapy for CIN 2-3.

\begin{tabular}{|c|c|c|}
\hline & Sensitivity & Specificity \\
\hline hr HPV-DNA & $95 \%$ & $75 \%$ \\
\hline Cytology & $70 \%$ & $78 \%$ \\
\hline Cotesting & $96 \%$ & $81 \%$ \\
\hline
\end{tabular}


Moreover, it has been observed that during post-treatment follow-up, hrHPV-DNA positivity is reduced from $90 \%$ to $20 \%$ ( $p<0.01$ ) at six months and positive endocervical margin of the cone, lesion grade in the surgical sample and age above 35 years have been indicated as significant independent factors predicting HPV persistence $[3,10]$. Furthermore, it has been observed that in a three-year follow-up period, HPV DNA persistence strongly correlates with residual/recurrent CIN and no women with type-specific HPV-negative had recurrent disease; on the contrary recurrences are persistently positive with the same genotype. In addition, it has been reported that $90 \%$ of recurrent CIN2-3 cases are infected by the same hrHPV type as before the initial treatment, conversely no recurrent disease has been found in HPV negative cases. Recent observations underline that residual or recurrent disease in women with persistent HPV16 and/or HPV18 is higher (82\%) than in women with persistence of other hrHPV types such as HPV 31, 33, 35, 45, 52 , and $58(66.7 \%)$ or HPV $39,51,56,59,68,26,53,66,73$, and $82(14.3 \%)[12,13]$. These data suggest different risk levels for the progression of CIN.

\section{HPV genotyping and risk stratification}

Despite the higher sensitivity, hrHPV testing shows a lower specificity than cytology (ratio $0.96,95 \% \mathrm{Cl}$ : 0.91-1.01) [1, 2] in identifying persistent disease or relapse since eradication of the clinical lesion does not necessarily mean eradication of all the infected tissue. Hence treated women may remain still positive for the virus as hrHPV testing does not distinguish between a persistent infection and a new transient one [7, 10].

As mentioned before, it is noted that among the women resulting HPV-positive during surveillance, certain genotypes confer a higher risk of post-therapy recurrence $[12,14]$. Hence persistence of some hrHPV types during the follow-up is associated with higher recurrence risk and warrant specific detection. These observations were confirmed by a recent report in which no recurrent or residual disease was found among women with type change or fluctuating HPV positivity, thus emphasising the importance of type-specific genotyping determination after treatment [13]. Nevertheless, data from a recent systematic review [15] highlighted that new HPV infections are a potential source of future disease following treatment, thus the clinical question is whether the persistence is related to the same original genotype, or belongs to a new infection.

As HPV genotyping assays become increasingly used in clinical practice, further research will be necessary to shed light on this controversial topic to determine whether type-specific HPV DNA tests can better define the risk stratification of disease persistence or recurrence and the length of the follow-up period in women harbouring specific hrHPV genotype [10].

Moreover recent studies suggest that the oncogenic activity of hrHPV-mRNA transcripts [16] may be a better indicator of women at risk for persistent disease or relapse showing a higher specificity and NPV than DNA based assay [17], while other reports point out that HPV E6/E7 mRNA is not useful for detecting relapse [18]; therefore more consistent data are necessary in order to delineate the usefulness of hrHPV-mRNA in clinical practice.

In conclusion, the role of hr-HPV-testing has been confirmed as an accurate index of disease clearance and is accepted along with Pap smear, in the routine post-treatment workup of CIN- treated patients. However, it is essential to make the distinction between individuals carrying the virus and those having a persistent clinical HPV lesion. The former are women in whom the only evidence for virus persistence can be obtained by the molecular diagnostic tools, but who do not have any clinically detectable HPV lesion whereas the latter group have a clinical lesion detectable by Pap smear, colposcopy, and biopsy. These are the cases that make the Pap test more specific and give it a higher positive predictive value (PPV) in detecting significant pathology compared with molecular tests [10].

Hence, it is clear that evidence-based post-treatment follow-up should include both conventional cytology and hrHPV-DNA testing to identify patients at increased risk for disease recurrence. 


\section{HPV-DNA testing after conservative treatment for adenocarcinoma in situ (AIS) lesions}

Over the past 50 years the incidence of invasive and pre-invasive glandular lesions of the uterine cervix has increased in western countries. Prior to 1970 adenocarcinomas accounted for approximately $5 \%$ of all cervical cancer cases, while by 1980 s they had increased to $20-25 \%$ of all cervical carcinomas [20]. Conservative surgery is the proposed treatment for these tumours in young women who desire to preserve their fertility. Nevertheless, the management of adenocarcinoma in situ (AIS) continues to be a controversial issue [21, 22]. In a review of 14 studies comprising 157 AIS patients with negative conisation margins, $26 \%$ harboured residual AIS and in $2 \%$ a unsuspected invasive cancer was discovered, implicating hysterectomy as the definitive treatment [23]. The conservative alternative to a hysterectomy is conisation with close surveillance, conventionally employing repeat cytology, colposcopy, and eventually punch biopsy and endocervical curettage. Unfortunately, these methods have a substantial false negative rate for glandular lesions both in primary diagnosis and in the follow-up of treated patients [24]. More recent data have indicated that hrHPV-DNA testing predicted residual/recurrent AIS or invasive adenocarcinoma (AdCa) during the follow-up of conservatively treated women for AIS significantly better than cytology, whose predictive power did not reach statistical significance at any of the follow-up visits. As shown in Table 2, hrHPV-DNA showed a better sensitivity than cytology (90\% versus $60 \%$ respectively at first follow-up visit), NPV (88\% versus $73 \%$ at first follow-up visit and $91 \%$ verus $87 \%$ at second follow-up visit respectively), while cytology was more specific in detecting residual AIS $(69 \%$ versus $58 \%$ at first follow-up visit and $73 \%$ versus $59 \%$ at second follow-up visit respectively); the combination of viral DNA testing and cytology reached $90 \%$ sensitivity in detecting persistent lesions at the first follow-up visit and $100 \%$ sensitivity at the second follow-up visit [24, 25].

Moreover, testing HR-HPV positive at any time during follow-up is the most significant independent predictor of recurrent disease. Finally, hrHPV detection was the single most powerful predictor of progression to invasive cancer. The status of the cone margins is also a good predictor and free cone margins had a significant protective effect against progressive disease [25].

These results suggest that testing hrHPV positive at any time point during follow-up is the most significant independent predictor of progressive disease, while showing free margins in cone has a significant protective effect against progression of invasive adenocarcinoma (AdCa). However, as a small percentage of conservatively treated AIS patients with persistent, recurrent, or progressive disease experienced a late (fifth and sixth FU visit) diagnosis of in situ or microinvasive AdCa, these women should be kept under close observation for at least the first three years after treatment.

Table 2. Performance of hrHPV-DNA testing, cytology and cotesting according to the follow-up visit of conservatively treated AIS patients.

\begin{tabular}{|l|c|c|c|}
\hline \multicolumn{5}{|l|}{ hrHPV DNA (\%) } & Cytology (\%) & Cotesting (\%) \\
\hline First FU visit & \multicolumn{5}{l|}{} \\
\hline Sensitivity & 90 & 60 & 90 \\
\hline Specificity & 58 & 69 & 50 \\
\hline PPV & 64 & 55 & 52 \\
\hline NPV & 88 & 73 & 89 \\
\hline Second FU visit & \multicolumn{5}{|l|}{} \\
\hline Sensitivity & 84 & 66 & 100 \\
\hline Specificity & 59 & 73 & 52 \\
\hline PPV & 42 & 44 & 40 \\
\hline NPV & 91 & 87 & 100 \\
\hline
\end{tabular}

AIS: Adenocarcinoma in situ, PPV: positive predictive value, NPV: negative predictive value. 


\section{Microinvasive squamosus cancer}

Microinvasive squamous cervical carcinoma (MIC), which most commonly occurs in young women who are of childbearing age is increasingly treated with conservative therapies in order to preserve fertility. As a consequence, an effective strategy allowing early detection of residual or progressive disease has become more and more important in post-treatment follow-up. To date, however, there are no standard practice guidelines on the optimal methods of surveillance of patients. Studies on long term follow-up after a primary conservative treatment have demonstrated that about $20 \%$ of patients experienced early or late diagnosis of recurrent disease; the majority (15\%) of cases had an intraepithelial lesion while in 5\% a MIC or invasive carcinoma was identified [26-28].

Follow-up protocols have so far consisted of repeat cytology and possibly colposcopy. The Pap smear has some inherent flaws, in particular a relatively high false negative rate, and the colposcopy has been shown to add very little to the detection rate of residual/ recurrent CIN [29]. Despite the increasing interest in the clinical uses of HPV testing, few follow-up studies of patients conservatively treated for stage IA have been performed, supporting the thesis that successful local treatment is accompanied by disappearance of HPV [30, 31]. Nevertheless, the scant available data have provided little advancement in knowledge of mechanisms of HPV clearance after conservative treatment of stage IA squamous cancer. In our institutions, a study is ongoing to follow up young patients treated by conisation using hrHPV-DNA testing and cytology in order to shed light on this new and interesting field. Primary results seem to indicate that the median time to viral clearance is relatively longer compared with patients treated for $\mathrm{CIN}$ and suggest a prolonged surveillance in these patients. However, the potential clinical value of HPV-DNA testing in this clinical setting needs to be confirmed by further observations.

\section{Conclusions}

A large number of studies have definitely demonstrated the excellent performance of hrHPV-DNA testing in the follow-up of conservative CIN 2-3/AIS treated patients. Possible options in the management of patients are indicated in Figure 1. Because of its sensitivity, negative predictive value, and optimal reproducibility, it is currently considered a powerful tool in the clinicians' hands to better manage post-treated patients for cervical squamous lesion or in situ adenocarcinoma.

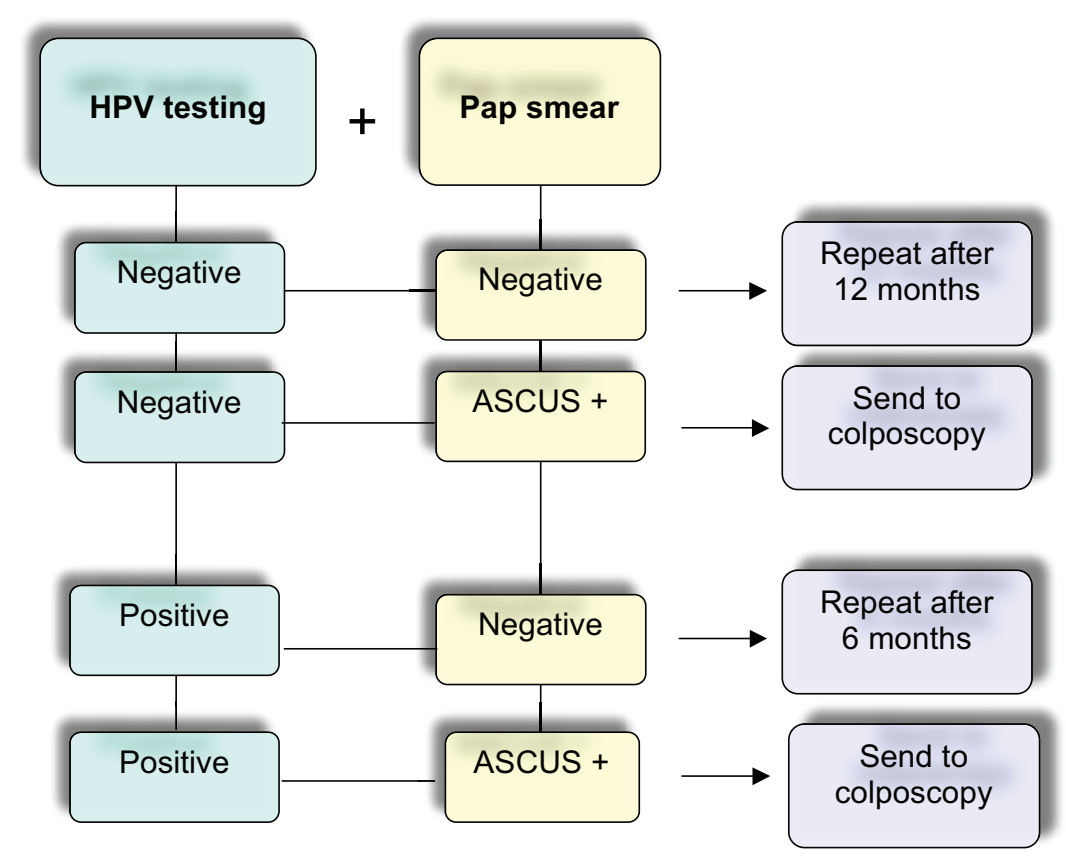

Figure 1. Flow chart of possible management using cotesting for cytology and hrHPV DNA in the follow-up after conservative treatment of high-grade squamous cervical lesions and adenocarcinoma in situ (AIS) [1, 2, 6, 10, 24]. 


\section{Acknowledgment}

This work is dedicated to our friend and colleague Mario Sideri. Mario was deeply involved in improving clinical procedure in early diagnosis and prevention of gynaecological malignancies, and his loss is intensely felt.

\section{References}

1. Paraskevaidis E et al (2001) The role of HPV DNA testing in the follow-up period after treatment for CIN: a systematic review of the literature Cancer Treat Rev 30(2) 205-211 DOI: 10.1016/j.ctrv.2003.07.008

2. Arbyn M et al (2006) Chapter 9: Clinical applications of HPV testing: A summary of meta-analyses Vaccine 24(Suppl 3) S3/78-89 DOI: $10.1016 /$ j.vaccine.2006.05.117

3. Sarian LO et al (2004) Factors associated with HPV persistence after treatment for high-grade cervical intra-epithelial neoplasia with large loop excision of the transformation zone (LLETZ) J Clin Virol 31(4) 270-4 DOI: 10.1016/j.jcv.2004.05.012 PMID: 15494268

4. Zielinski GD et al (2004) HPV testing and monitoring of women after treatment of CIN 3: review of the literature and metaanalysis Obstet Gynecol Surv 59(7) 543-53 DOI: 10.1097/00006254-200407000-00024 PMID: 15199273

5. Melnikow J et al (2009) Cervical intraepithelial neoplasia outcomes after treatment: long-term follow-up from the British Columbia cohort study J Natl Cancer Inst 101 721-8 DOI: 10.1093/jnci/djp089 PMID: 19436026 PMCID: 2684554

6. Costa S et al (2002) Disease persistence in patients with cervical intraepithelial neoplasia undergoing electrosurgical conization Gynecol Oncol 85(1) 119-24 DOI: 10.1006/gyno.2001.6579 PMID: 11925130

7. Kocken $\mathrm{M}$ et al (2011) Risk of recurrent high-grade cervical intraepithelial neoplasia after successful treatment: a long-term multi-cohort study Lancet Oncol 12(5) 441-50 DOI: 10.1016/S1470-2045(11)70078-X PMID: 21530398

8. Soutter WP, Sasieni P and Paraskevaidis E (2006) Long-term risk of invasive cervical cancer after treatment of squamous cervical intraepithelial neoplasia Int J Cancer 118(8) 2048-55 DOI: 10.1002/ijc.21604

9. J Jordan P et al (2009) European guidelines for clinical management of abnormal cervical cytology, Part 2 Cytopathology 20(1) 5-16 DOI: 10.1111/j.1365-2303.2008.00636.x

10. Costa S et al (2003) Factors predicting human papillomavirus clearance in cervical intraepithelial neoplasia lesions treated by conization Gynecol Oncol 90(2) 358-65 DOI: 10.1016/S0090-8258(03)00268-3 PMID: 12893200

11. Massad LS et al (2012) ASCCP Consensus Guidelines Conference 2012 updated consensus guidelines for the management of abnormal cervical cancer screening tests and cancer precursors Obstet Gynecol 121(4) 829-46

12. Venturoli S et al (2008) Correlation of high-risk human papillomavirus genotypes persistence and risk of residual or recurrent cervical disease after surgical treatment J Med Virol 80(8) 1434-40 DOI: 10.1002/jmv.21198 PMID: 18551620

13. Origoni M et al (2012) HPV-DNA testing for cervical cancer precursors: from evidence to clinical practice Ecancermedicalscience 6258 PMID: 22778786 PMCID: $\underline{3388143}$

14. Soderlund-Strand A, Kjellberg L and Dillner J (2014) Human Papillomavirus Type-Specific Persistence and Recurrence After Treatment for Cervical Dysplasia J Med Virol 86(4) 634-64 DOI: 10.1002/jmv.23806

15. Rositch AF et al (2014) The incidence of human papillomavirus infection following treatment for cervical neoplasia: a systematic review Gynecol Oncol 132(3) 767-79 DOI: 10.1016/j.ygyno.2013.12.040 PMID: 24412508 
16. M Cricca et al (2007) Viral DNA load, physical status and E2/E6 ratio as markers to grade HPV16 positive women for high-grade cervical lesions Gynecol Oncol 106(3) 549-57 DOI: 10.1016/j.ygyno.2007.05.004 PMID: 17568661

17. Frega A et al (2014) Assessment of HPV-mRNA test to predict recurrent disease in patients previously treated for CIN 2/3 J Clin Virol 60(1) 39-43 DOI: 10.1016/j.jcv.2014.01.017 PMID: 24602516

18. EA Burger et al (2011) HPV mRNA tests for the detection of cervical intraepithelial neoplasia: a systematic review Gynecol Oncol 120(3) 430-8 DOI: 10.1016/j.ygyno.2010.11.013

19. Arbyn $\mathrm{M}$ et al (2005) Clinical utility of HPV-DNA detection: triage of minor cervical lesions, follow-up of women treated for high-grade CIN: an update of pooled evidence Gynecol Oncol 99(3 Suppl 1) 7-11 DOI: 10.1016/j.ygyno.2005.07.033

20. Smith $\mathrm{HO}$ et al (2000) The rising incidence of adenocarcinoma relative to squamous cell carcinoma of the uterine cervix in the US. A 24-year population based study Gynecol Oncol 78 97-105 DOI: 10.1006/gyno.2000.5826 PMID: 10926787

21. Bryson $P$ et al (2004) Is electrosurgical loop excision with negative margins sufficient treatment for cervical ACIS? Gynecol Oncol 93(2) 465-8 DOI: 10.1016/j.ygyno.2004.01.028 PMID: 15099963

22. American College of Obstetricians and Gynecologists (2008) ACOG Practice Bulletin No. 99 (2008): management of abnormal cervical cytology and histology Obstet Gynecol 112(6) 1419-44 DOI: 10.1097/AOG.0b013e318192497c PMID: 19037054

23. Cohn DE et al (2005) Invasive cervical adenocarcinoma immediately following a cone biopsy for adenocarcinoma in situ with negative margins Gynecol Oncol 98(1) 158-60 DOI: 10.1016/j.ygyno.2005.04.013 PMID: 15913738

24. Costa $\mathrm{S}$ et al (2007) Human papillomavirus (HPV) test and PAP smear as predictors of outcome in conservatively treated adenocarcinoma in situ (AIS) of the uterine cervix Gynecol Oncol 106(1) 170-6 DOI: 10.1016/j.ygyno.2007.03.016 PMID: 17481701

25. Costa $S$ et al (2012) Factors predicting the outcome of conservatively treated adenocarcinoma in situ of the uterine cervix: an analysis of 166 cases Gynecol Oncol 124(3) 490-5 DOI: 10.1016/j.ygyno.2011.11.039

26. Kolstad $P$ (1989) Follow up study of 232 patients with stage $1 \mathrm{~A} 1$ and 411 patients with stage $1 \mathrm{~A} 2$ squamous cell carcinoma of the cervix Gynaecol Oncol 33(3) 265-72 DOI: 10.1016/0090-8258(89)90510-6

27. Costa S et al (2009) Outcome of Conservatively Treated Microinvasive Squamous Cell Carcinoma of the Uterine Cervix During a 10-Year Follow-up Int J Gynecol Cancer 19(1) 33-38 DOI: 10.1111/IGC.0b013e318197f53b PMID: 19258938

28. Tseng C, Liang C and Lin C (1999) A study of diagnostic failure of loop conization in microinvasive carcinoma of the cervix Gynecol Oncol 73(1) 91-5 DOI: 10.1006/gyno.1998.5295 PMID: 10094886

29. Orlandi $C$ et al (1995) Presurgical assessment and therapy of microinvasive carcinoma of the cervix Gynecol Oncol 59(2) 255-60 DOI: $\underline{10.1006 / g y n o .1995 .0018}$ PMID: 7590483

30. Cairns M et al (2010) High-risk HPV genotyping in the follow-up of women treated conservatively for microinvasive cervical cancer Int J Gynecol Cancer 20(1) 154-7 DOI: 10.1111/IGC.0b013e3181c3a6b6 PMID: 20130517

31. Korolenkova LI (2011) Value of HPV (hybrid capture 2) testing for assessing the effectiveness of conservative surgical treatment in CIN2-3/carcinoma in situ and microinvasive cervical cancer Vopr Onkol 57(3) 322-6 PMID: 21882603 